\title{
Prevalence of Syphilis among Blood and Stem Cell Donors in Saudi Arabia: An Institutional Experience
}

Ghaleb Elyamany ${ }^{1,2}$, Mohamed Al amro ${ }^{1}$, Winston Costa Pereira ${ }^{1}$, Omar Alsuhaibani ${ }^{1}$

${ }^{1}$ Department of Central Military Laboratory and Blood Bank, Prince Sultan Military Medical City, Riyadh, Saudi Arabia

${ }^{2}$ Department of Hematology, Theodor Bilharz Research Institute, Egypt

\section{Type of article: Original}

\begin{abstract}
Introduction: Syphilis is one of the known transfusion-transmissible infections and causes 100,000 deaths yearly, with around $90 \%$ of these deaths occurring in the developing world. Little data is available regarding the prevalence of syphilis among Saudi blood and stem cell donors. We conducted a survey on the incidence of syphilis among all blood and stem cell donors.

Methods: This study was conducted at the Prince Sultan Military Medical City in Riyadh, Saudi Arabia in the 10 years period data during 2006-2015. Data were analyzed about full history, physical examination, age, sex, weight, profession, marital status, number of the donations, data of last donation, having a relation who received blood transfusion, as well as the screening test results of the donated blood. We determined the seroprevalence of infection and compared by sex and other variable through frequency analysis, Chi square, Fisher, and prevalence ratios.

Results: Approximately 240,000 blood donors were screened and studied in the period of study. Most of the blood donors were male (98.3\%) and $89 \%$ of them were citizens of Saudi Arabia. According to our findings, we estimated that, in the last 10 years, approximately $0.044 \%$ of all the blood donors were syphilis positive cases. No cases were detected as positive for syphilis among stem cell donors. Only 60 blood donors tested positive for syphilis. In addition, we studied 202 stem cell transplant donors during the same period, of which $59 \%$ were male and none texted positive for syphilis.

Conclusions: A concerted effort between the government, health care providers, regulatory bodies and accreditation agencies have all contributed in eliminating the risk of spreading syphilis among blood donors.

Keywords: Transfusion-transmissible infection, Blood Donor, Stem cell donor, Saudi Arabia, Syphilis
\end{abstract}

\section{Introduction}

Blood donation is an important procedure that saves millions of lives; however, unsafe transfusion practices carry the risk of transfusion-transmissible infections (TTIs) (1). An unsafe blood transfusion is very costly from both an economic and a human point of view, not only for the recipients themselves, but also for their families and their communities $(2,3)$. Syphilis is caused by the spirochete bacterium Treponema pallidum, which is known to be transmitted sexually and from mother to child. This bacterium is known to infect any organ (4). Clinical manifestation of this disease, when transmitted from mother to child, include spontaneous abortion, stillbirth, manifestation of congenital syphilis, infant death, and late sequelae (5). Syphilis is a chronic disease that occurs worldwide and its prevalence varies significantly in different geographic locations. This disease was first discovered in 1905 and has been mainly described as a sexually transmitted disease. The World Health Organization (WHO) has conducted a survey on the prevalence of four sexually transmitted diseases: chlamydia trachomatis, neisseria gonorrhoeae, syphilis, and trichomonas vaginalis. It was found that syphilis accounts for approximately $10 \%$ of these sexually transmitted diseases. Another common route of transmission is through the placenta, thus screening for syphilis is mandatory for umbilical cord blood donors. Transmission of syphilis by means other than the sexual

\section{Corresponding author:}

Dr. Ghaleb Elyamany, Department of Central Military Laboratory and Blood Bank, Prince Sultan Military Medical City, Riyadh, Saudi Arabia. Tel: +966569647181, Email: ghalebelyamany@yahoo.com

Received: June 02, 2016, Accepted: July 12, 2016, Published: August 2016 iThenticate screening: July 12, 2016, English editing: July 20, 2016, Quality control: July 28, 2016

(C) 2016 The Authors. This is an open access article under the terms of the Creative Commons Attribution-NonCommercialNoDerivs License, which permits use and distribution in any medium, provided the original work is properly cited, the use is non-commercial and no modifications or adaptations are made. 
route is infrequent (6). There is no documented evidence to show that syphilis is transmitted via organ transplantation. However, serologic testing of organ donors for syphilis is recommended, but the presence of donor syphilis infection (past or present) is not considered a contraindicator for transplantation if prophylactic antibiotics are administered to the recipient (7). In this study, we conducted a survey on the incidence of syphilis among all blood and stem cell donors at the Prince Sultan Military Medical City (PSMMC) in Riyadh, Saudi Arabia (SA) during a 10 years period from 2006 to 2015 .

\section{Material and Methods}

We have conducted a survey on all the blood and stem cell donors at the Prince Sultan Military Medical City (PSMMC) in Riyadh, Saudi Arabia. The total number of blood donors that were screened during the last ten-year is 239,330. The male donors consisted of a large group of 235,346 and 3,984 were female donors. A full history and physical examination were performed and recorded for all volunteer blood donors to review their eligibility for donation. The data included age, sex, weight, profession, marital status, being a relative of the patient receiving the transfusion or a voluntary donor, donation number, date of the last donation, information of received blood quantity and type, information of screening test results, and other relevant data. Also, the physical examination and laboratory findings were recorded. Similarly for stem cell donors, a full history and physical examination were performed and recorded for all donors to review their eligibility for donation. We use stem cells collected BY apheresis either from autologous patients or allogeneic donors. We screened 202 donors from 2006 until 2015 . The blood tests that were conducted on potential donors included the treponemal test (Roche Diagnostics), which uses total antibodies to detect Treponema Pallidum (TP). These tests detect specific antitreponemal antibodies to antigens, indicating exposure during the patient's life. A positive test is an indicator of syphilis, which may be in a latent stage for years or decades. This test is specific, but may report false positive cases especially in low prevalence populations. In summary, this test uses the sandwich principle of immunoassays for an in vitro qualitative determination of total antibodies to TP in human serum and plasma. TP detection was confirmed by using the IgM-class of antibodies to TP in the human serum. The DIAsource KAPRSPM17 Syphilis IgM Elisa kit was used as a part of the confirmatory test. Briefly, in this assay, controls and unknowns were incubated in microtitration wells coated with purified and inactivated treponema antigen. After incubation and washing, the wells are treated with a conjugate composed of anti-human IgM antibodies labeled with the substrate tetramethylbenzidine (TMB). At the end of the test, the absorbance is measured at a wavelength of $450 \mathrm{~nm}$. We determined the seroprevalence of infection and compared using sex and other variable through frequency analysis, chi square, Fisher, and prevalence ratios.

\section{Results}

The total number of blood donors screened was 239,330, of which male donors consisted of the majority of 235,346 $(98.3 \%)$ and the female donors were 3,984 (1.7\%). In this study, 89\% of blood donors (both male and female) were Saudis. The mean and median age of all blood donors (both male and female) was 36 and 35, respectively. The prevalence of syphilis is shown in Table 1.

Table 1. The total number of blood donors screened for syphilis within the last 10 years. The positive, negative, and equivocal cases are shown.

\begin{tabular}{|l|l|l|l|}
\hline Donors Syphilis Screening $(2006-2015)$ & \\
\hline Group & Total & Male & Female \\
\hline Total Screened Donors & 239,330 & 235,346 & 3,984 \\
\hline Positive cases & $60(0.025 \%)$ & $57(0.024 \%)$ & $3(0.075 \%)$ \\
\hline Equivocal & $46(0.019 \%)$ & $45(0.019 \%)$ & $1(0.075 \%)$ \\
\hline Negative cases & 239,224 & 235,244 & 3,980 \\
\hline Mean Age (year) & 36 & 36 & 35 \\
\hline Median Age (year) & 35 & 35 & 33 \\
\hline
\end{tabular}

The prevalence of positive and equivocal results for syphilis was $0.044 \%$ of the total population of blood donors screened. This study has shown that only 60 blood donors were positive among the 239,330 donors screened, which amounts to a mere $0.025 \%$. Among positive cases, only 3 donors were female, which amounted to $0.075 \%$. We tested another group, which was ambiguous in nature, and the positive results for this group could not be conclusively determined. This group is referred to as the equivocal group. We had 46 donors in this group whose blood donations were differed is a reproducible manner, out of which 45 were male donors. Regarding the age 
groups of the positive and equivocal cases, we assessed the male group due to the very low numbers in the female group. We have seen that the highest number of positive cases lie between the ages of 21-30 years, whereas the highest number of equivocal cases lie within the 41-50 age group (Figure 1). Overall, we have a very small percentage of positive or equivocal cases of syphilis, whereas a majority of donors were shown to have negative results in both the male and female groups. Similarly, out of 202 cases of stem cell transplants donors, male donors were $119(59 \%)$ and female donors were $83(41 \%)$. The mean age for all donors was 30 years (Table 2). We have not detect any positive case of syphilis among the stem cell donors $(0 \%)$ in our study.

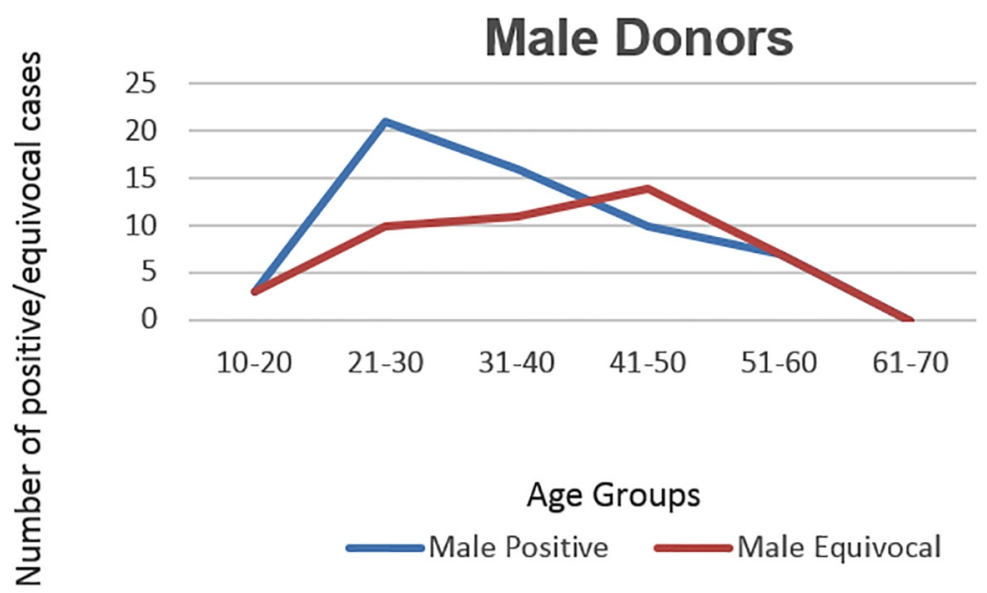

Figure 1. Incidence of syphilis in different age groups among male donors.

Table 2. The total number of stem cell donors screened for syphilis from 2001 until 2015. The positive and negative cases are shown.

\begin{tabular}{|l|l|l|l|}
\hline Stem Cell Donors Syphilis Screening (2001- 2015 \\
\hline Group & Total & Male & Female \\
\hline Total Screened Donors & 202 & 119 & 83 \\
\hline Positive cases & 0 & 0 & 0 \\
\hline Negative cases & 202 & 119 & 83 \\
\hline Mean Age (year) & 30 & 32 & 28 \\
\hline Median Age (year) & 29 & 31 & 26 \\
\hline
\end{tabular}

\section{Discussion and conclusions}

Syphilis can be transmitted during direct sexual contact, through blood transfusions, and from a pregnant woman to her unborn child (8). However, blood transfusion currently play a minor importance due to the available sensitive methods of detection, as well as routine screening of blood products for syphilis. Screening for syphilis is mandatory for routine blood transfusions, as well as cord blood donations. There are currently 36 million people infected with syphilis worldwide, with 12 million new cases reported every year. The WHO prepared a report in 2008 on the global incidence and prevalence of selected curable sexually transmitted infections. Although, most new syphilis cases occur in developing countries, syphilis has re-emerged as a health concern in a number of developed nations over the past decade (9). A 5-year surveillance study has been conducted to assess and identify the prevalence of sexually transmitted disease in Saudi Arabia (SA) (10). It should be noted that there is an annual incidence of approximately 3,500 cases sexually transmitted cases in SA, of which the percentage of syphilis accounts for $8.7 \%$. This is marginally lower than the global average of $10 \%$ of sexually transmitted diseases (11). In the current study, we have seen that the highest number of positive cases are aged between 21-30 years, whereas the highest number of equivocal cases lie within the 41-50 age group. This data is consistent with a global study that showed a high prevalence of positive cases among young donors $(12,13)$. In our study, the prevalence of positive and equivocal cases of syphilis was $0.044 \%$ of the total population of blood donors screened. This very small percentage is due to a stringent screening and the efforts of local government agencies, regulatory, and accrediting agencies focused on the elimination of syphilis and spreading awareness among individuals. Similar or very near results were reported in other countries; $0 \%$ seroprevalence among Iranian and Egyptian blood donors $(14,15), 0.02 \%$ in Turkey (16), 0.1\% reported in Port Harcourt, $0.2 \%$ among blood donors in the Niger delta of Nigeria $(17,18)$, and $0.75 \%$ in Pakistan (19); whereas higher results were reported in Ghana (7.5\%) (20), in Ethiopia (12.8\%) (21), in Tanzania (12.7\%) 
(22), and in Nigeria (3.6\%) (23). In our institute, we use stem cells collected using apheresis either from autologous patients or allogeneic donors. We have not seen any case of syphilis among stem cell donors due to strict regulatory requirements. In our transfusion facility we follow the guidelines of international accreditation agencies, such as the American Association of Blood Banks (AABB) and the Foundation for Accreditation for Cellular Therapy (FACT), as well as the Joint Accreditation Committee for ISCT and EBMT (JACIE). The guidelines stipulated by these organizations have greatly assisted the containment of syphilis in our community. However, in the years to come, we hope to eradicate syphilis entirely through a concerted effort with the WHO, local government agencies, regulatory and accrediting agencies through the elimination of syphilis and spreading awareness among individuals in the community.

\section{Acknowledgments:}

The authors wish to thank the Prince Sultan Military Medical City in Riyadh (Saudi Arabia) for supporting this study.

\section{Conflict of Interest:}

There is no conflict of interest to be declared.

Authors' contributions:

All authors contributed to this project and article equally. All authors read and approved the final manuscript.

\section{References:}

1) Diro E, Alemu S, G/Yohannes A. Blood safety \& prevalence of transfussion transmissible viral infections among donors at the Red Cross Blood Bank in Gondar University Hospital. Ethiop Med J. 2008; 46(1): 7 13. PMID: 18711984.

2) Tessema B, Yismaw G, Kassu A, Amsalu A, Mulu A, Emmrich F, et al. Seroprevalence of HIV, HBV, $\mathrm{HCV}$ and syphilis infections among blood donors at Gondar University Teaching Hospital, Northwest Ethiopia: declining trends over a period of five years. BMC Infect Dis. 2010; 10: 111. doi: 10.1186/1471 2334-10-111. PMID: 20459703, PMCID: PMC2881920.

3) Nada HA, Atwa M. Seroprevalence of HBV, HCV, HIV and Syphilis Markers among Blood Donors at Suez Canal University Hospital Blood Bank. J Blood Disord Transfus. 2013; 5: 177. doi: 10.4172/21559864.1000177.

4) Nyatsanza F, Tipple C. Syphilis: presentations in general medicine. Clin Med (Lond). 2016; 16(2): 184-8. doi: 10.7861/clinmedicine.16-2-184. PMID: 27037391.

5) Eleonor GL. Current Perspective on Prevention of Mother-to-Child Transmission of Syphilis. Cureus. 2016; 8(3): 525. doi: 10.7759/cureus.525. PMID: 27081586, PMCID: PMC4829408.

6) Singh AE, Romanowski B. Syphilis: review with emphasis on clinical, epidemiologic, and some biologic features. Clin Microbiol Rev. 1999; 12(2): 187-209.

7) Fischer SA, Avery RK; AST Infectious Disease Community of Practice. Screening of donor and recipient prior to solid organ transplantation. Am J Transplant. 2009; 9 Suppl 4: 7-18. doi: 10.1111/j.16006143.2009.02888.x. PMID: 20070698.

8) Kim JK, Choi SR, Lee HJ, Kim DH, Yoon MS, Jo HS. Conngenital syphilis presenting with a generalized bullous and pustular eruption in a premature newborn. Ann Dermatol. 2011; 23 Suppl 1: 127-30. doi: 10.5021/ad.2011.23.S1.S127. PMID: 22028559, PMCID: PMC3199409.

9) World Health Organization. Global incidence and prevalence of selected curable sexually transmitted infections-2008. (Geneva: WHO). 2012; 20.

10) Madani TA. Sexually transmitted infections in Saudi Arabia. BMC Infec Dis. 2006; 6: 3. doi: 10.1186/1471-2334-6-3.

11) Nakashima AK, Rolfs RT, Flock ML, Kilmarx P, Greenspan JR. Epidemiology of syphilis in the United States, 1941-1993. Sex Transm Dis. 1996, 23(1): 16-23. PMID: 8801638.

12) French P. Syphilis. BMJ. 2007; 334(7585): 143-7. doi: 10.1136/bmj.39085.518148.BE. PMID: 17235095, PMCID: PMC1779891.

13) Centers for Disease Control and Prevention. STD surveillance 2011. Atlanta. 2012.

14) Samkange-Zeeb FN, Spallek L, Zeeb H. Awareness and knowledge of sexually transmitted diseases (STDs) among school-going adolescents in Europe: a systematic review of published literature. BMC Public Health. 2011; 11: 727. doi: 10.1186/1471-2458-11-727. PMID: 21943100, PMCID: PMC3189891. 
15) Nada HA, Atwa M. Seroprevalence of HBV, HCV, HIV and Syphilis Markers among Blood Donors at Suez Canal University Hospital Blood Bank. J Blood Disord Transfus. 2013; 5: 177. doi: 10.4172/21559864.1000177.

16) Zohreh A, Mazyar G, Bashir H, Sherun A, Seyed MA. Zero prevalence of syphilis among blood donors in Tehran, Iran. Transf Today. 2005; 64: 24.

17) Oner S, Yapici G, Sasmaz CT, Kurt AO, Bugdayci R. Hepatitis B, hepatitis C, HIV, and VDRL seroprevalence of blood donors in Mersin, Turkey. Turk J Med Sci. 2011; 41(2): 335-41. doi: 10.3906/sag0906-64.

18) Ejele OA, Erhabor O, Nwauche CA. Trends in the prevalence of some transfusion-transmissible infections among blood donors in Port Harcourt, Nigeria. Haema. 2004; 8(2): 237-73.

19) Erhabor O, Nwoka E, Adias TC. Seroprevalence of Treponema palladium infection among blood donors in a resource-poor setting in the Niger Delta of Nigeria. Africa Sanguine. 2007; 10: 19-21.

20) Bhatti FA, Ullah Z, Salamat N, Ayub M, Ghani E. Anti-hepatits B core antigen testing, viral markers, and occult hepatitis B virus infection in Pakistani blood donors: implications for transfusion practice. Transfusion. 2007; 47(1): 74-9. doi: 10.1111/j.1537-2995.2007.01066.x. PMID: 17207233.

21) Adjei AA, Kudzi W, Armah H, Adiku T, Amoah AG, Ansah J. Prevalence of antibodies to syphilis among blood donors in Accra, Ghana. Jpn J Infect Dis. 2003; 56(4): 165-7. PMID: 14583641.

22) Bloch EM, Vermeulen M, Murphy E. Blood transfusion safety in Africa: a literature review of infectious disease and organizational challenges. Transfus Med Rev. 2012; 26(2): 164-80. doi: 10.1016/j.tmrv.2011.07.006. PMID: 21872426, PMCID: PMC3668661.

23) Matee MI, Magesa PM, Lyamuya EF. Seroprevalence of human immunodeficiency virus, hepatitis B and C viruses and syphilis infections among blood donors at the Muhimbili National Hospital in Dar es Salaam, Tanzania. BMC Public Health. 2006; 6: 21. doi: 10.1186/1471-2458-6-21. PMID: 16445860, PMCID: PMC1373616.

24) Chikwem JO, Mohammed I, Okara GC, Ukwandu NC, Ola TO. Prevalence of transmissible blood infections among blood donors at the University of Maiducuri Teaching Hospital, Maiduguri, Nigeria. East Afr Med J. 1997; 74(4): 213-6. PMID: 9299819. 